\title{
On the convergence of quasi-Random Sampling Importance Resampling
}

\author{
Bart Vandewoestyne* Ronald Cools
}

Department of Computer Science

K.U.Leuven, Belgium

June 21, 2007 


\section{Outline}

(1) Introduction

(2) Quasi-Monte Carlo tools

(3) The SIR and QSIR algorithm

(4) Numerical Example

(5) Conclusions 
On the convergence of quasi-Random Sampling Importance Resampling

Introduction

\section{Outline}

(1) Introduction

(2) Quasi-Monte Carlo tools

(3) The SIR and QSIR algorithm

(4) Numerical Example

(5) Conclusions 


\section{The problem}

The problem we study is ...

- ... the generation of samples from a given probability density function (PDF)

$$
f(\boldsymbol{\theta})=\frac{h(\boldsymbol{\theta})}{\int_{\mathbb{R}^{s}} h(\boldsymbol{\theta}) d \boldsymbol{\theta}} .
$$




\section{The problem}

The problem we study is ...

- ... the generation of samples from a given probability density function (PDF)

$$
f(\boldsymbol{\theta})=\frac{h(\boldsymbol{\theta})}{\int_{\mathbb{R}^{s}} h(\boldsymbol{\theta}) d \boldsymbol{\theta}} .
$$

- However, we do not know the normalizing constant:

$$
\int_{\mathbb{R}^{s}} h(\boldsymbol{\theta}) d \boldsymbol{\theta}=?
$$




\section{Generation of samples: normalized PDF}

If the normalizing constant is known, one can use for example the inverse CDF method ...

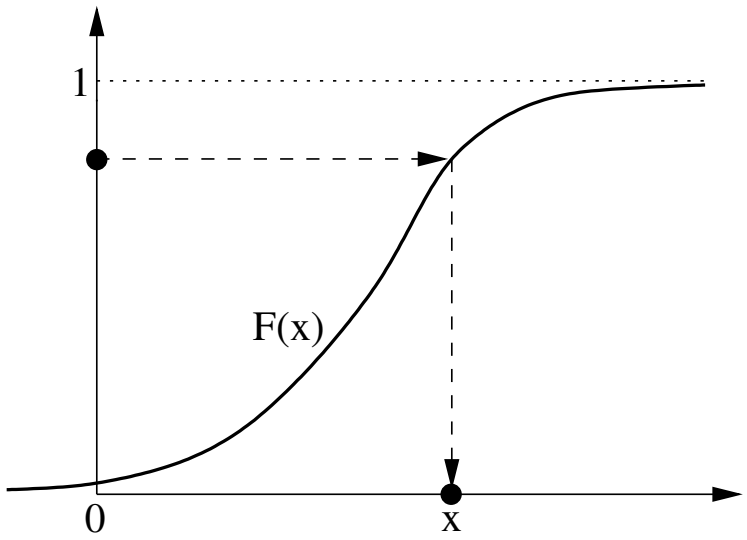




\section{Generation of samples: normalized PDF}

... or other well-known methods from the literature. See for example
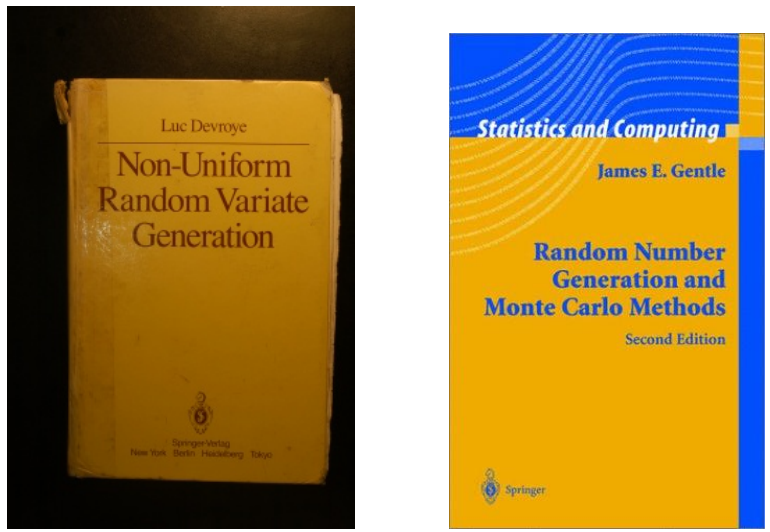
Generation of samples: normalizing constant unknown

Things become more complicated if the normalizing constant is not known:

$$
f(\boldsymbol{\theta})=\frac{h(\boldsymbol{\theta})}{\int_{\mathbb{R}^{s}} h(\boldsymbol{\theta}) d \boldsymbol{\theta}} .
$$

How can we do this? 
Generation of samples: normalizing constant unknown

Things become more complicated if the normalizing constant is not known:

$$
f(\boldsymbol{\theta})=\frac{h(\boldsymbol{\theta})}{\int_{\mathbb{R}^{s}} h(\boldsymbol{\theta}) d \boldsymbol{\theta}} .
$$

How can we do this?

- Sampling Importance Resampling (SIR) [Rubin, D. B., 1988] 
Generation of samples: normalizing constant unknown

Things become more complicated if the normalizing constant is not known:

$$
f(\boldsymbol{\theta})=\frac{h(\boldsymbol{\theta})}{\int_{\mathbb{R}^{s}} h(\boldsymbol{\theta}) d \boldsymbol{\theta}} .
$$

How can we do this?

- Sampling Importance Resampling (SIR) [Rubin, D. B., 1988]

- Quasi-Random Sampling Importance Resampling (QSIR) [Pérez et al., 2005] 
Generation of samples: normalizing constant unknown

Things become more complicated if the normalizing constant is not known:

$$
f(\boldsymbol{\theta})=\frac{h(\boldsymbol{\theta})}{\int_{\mathbb{R}^{s}} h(\boldsymbol{\theta}) d \boldsymbol{\theta}} .
$$

How can we do this?

- Sampling Importance Resampling (SIR) [Rubin, D. B., 1988]

- Quasi-Random Sampling Importance Resampling (QSIR) [Pérez et al., 2005]

$\Rightarrow$ We will take a closer look at QSIR. . 
But let's not rush and start with the start...

We need some quasi-Monte Carlo ideas and concepts.

Let's take a look at them... 
On the convergence of quasi-Random Sampling Importance Resampling

Quasi-Monte Carlo tools

\section{Outline}

\section{(1) Introduction}

(2) Quasi-Monte Carlo tools

(3) The SIR and QSIR algorithm

(4) Numerical Example 


\section{Koksma-Hlawka inequality}

\section{Theorem (Koksma-Hlawka)}

$$
\left|\frac{1}{N} \sum_{n=1}^{N} f\left(\mathbf{x}_{n}\right)-\int_{[0,1]^{s}} f(\mathbf{x}) d \mathbf{x}\right| \leq V_{H K}(f) D_{N}^{*}(\mathcal{P})
$$

- Well-known among MC and QMC-people.

- No further explanation needed here. 


\section{Discrepancy}

\section{Definition (Star-discrepancy of a point set $\mathcal{P}$ )}

$$
D_{N}^{*}(\mathcal{P}):=\sup _{\mathbf{x} \in[0,1]^{s}}\left|\frac{1}{N} \sum_{n=1}^{N} I_{[0, \mathbf{x})}\left(\mathbf{x}_{n}\right)-\operatorname{Vol}(\mathbf{x})\right|
$$

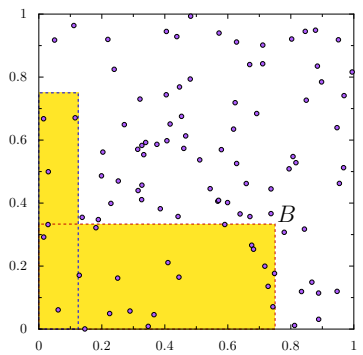

- Well-known among MC and QMC-people.

- No further explanation needed here. 


\section{Discrepancy in one-dimension}

\section{Theorem}

For a one-dimensional ordered point set $\mathcal{P}$, we have

$$
D_{N}^{*}(\mathcal{P})=\frac{1}{2 N}+\max _{1 \leq n \leq N}\left|x_{n}-\frac{2 n-1}{2 N}\right| .
$$

- This is for one-dimensional point sets.

- The point set must be ordered. 


\section{Discrepancy in one-dimension}

\section{Theorem}

For a one-dimensional ordered point set $\mathcal{P}$, we have

$$
D_{N}^{*}(\mathcal{P})=\frac{1}{2 N}+\max _{1 \leq n \leq N}\left|x_{n}-\frac{2 n-1}{2 N}\right| .
$$

\section{Corollary}

In one dimension, the point set

$$
x_{i}=\frac{2 i-1}{2 N}, \quad i=1, \ldots, N
$$

has the lowest possible discrepancy of $\frac{1}{2 N}$. 


\section{Our goal}

So far our first QMC-steps...

Let us now try to formalize what we are trying to accomplish:

- We are given ... 


\section{Our goal}

So far our first QMC-steps...

Let us now try to formalize what we are trying to accomplish:

- We are given ...

- ... a multidimensional point set, 


\section{Our goal}

So far our first QMC-steps...

Let us now try to formalize what we are trying to accomplish:

- We are given ...

- ... a multidimensional point set, - ... where each point can have an associated weight. 


\section{Our goal}

So far our first QMC-steps...

Let us now try to formalize what we are trying to accomplish:

- We are given ...

- ... a multidimensional point set,

- ... where each point can have an associated weight.

- We ask ourselves the questions: 


\section{Our goal}

So far our first QMC-steps...

Let us now try to formalize what we are trying to accomplish:

- We are given ...

- ... a multidimensional point set,

- ... where each point can have an associated weight.

- We ask ourselves the questions:

- How representative is this point set for a certain cumulative distribution function (CDF)? 


\section{Our goal}

So far our first QMC-steps. . .

Let us now try to formalize what we are trying to accomplish:

- We are given ...

- ... a multidimensional point set,

- ... where each point can have an associated weight.

- We ask ourselves the questions:

- How representative is this point set for a certain cumulative distribution function (CDF)?

- How do we measure 'representativeness' of a point set? 


\section{Measuring 'representativeness'}

We use the following definition for the Empirical Cumulative Distribution Function (ECDF):

- If weights are specified:

$$
F_{N}(\mathbf{x}):=\sum_{i=1}^{N} q_{i} l_{(-\infty, \mathbf{x}]}\left(\mathbf{x}_{i}\right) \quad \text { with } q_{i}>0 \text { and } \sum_{i=1}^{N} q_{i}=1 .
$$




\section{Measuring 'representativeness'}

We use the following definition for the Empirical Cumulative Distribution Function (ECDF):

- If weights are specified:

$$
F_{N}(\mathbf{x}):=\sum_{i=1}^{N} q_{i} l_{(-\infty, \mathbf{x}]}\left(\mathbf{x}_{i}\right) \quad \text { with } q_{i}>0 \text { and } \sum_{i=1}^{N} q_{i}=1 .
$$

- If no weights are specified, assume all weights uniform:

$$
F_{N}(\mathbf{x})=\frac{1}{N} \sum_{i=1}^{N} I_{(-\infty, \mathbf{x}]}\left(\mathbf{x}_{i}\right) .
$$


On the convergence of quasi-Random Sampling Importance Resampling

Quasi-Monte Carlo tools

\section{Measuring 'representativeness'}

How do we measure the "representativeness" of a point set $\mathcal{P}$ ? 


\section{Measuring 'representativeness'}

How do we measure the "representativeness" of a point set $\mathcal{P}$ ?

Definition (F-discrepancy, [Wang \& Fang, 1990])

$$
D_{N}^{*}(\mathcal{P}, F):=\sup _{\mathbf{x} \in \mathbb{R}^{s}}\left|F_{N}(\mathbf{x})-F(\mathbf{x})\right|
$$




\section{Measuring 'representativeness'}

How do we measure the "representativeness" of a point set $\mathcal{P}$ ?

Definition (F-discrepancy, [Wang \& Fang, 1990])

$$
D_{N}^{*}(\mathcal{P}, F):=\sup _{\mathbf{x} \in \mathbb{R}^{s}}\left|F_{N}(\mathbf{x})-F(\mathbf{x})\right|
$$

We speak of $F$-discrepancy...

- ... of a point set,

- ... with respect to $F$. 


\section{Measuring 'representativeness'}

Definition (F-discrepancy, [Wang \& Fang, 1990])

$$
D_{N}^{*}(\mathcal{P}, F):=\sup _{\mathbf{x} \in \mathbb{R}^{s}}\left|F_{N}(\mathbf{x})-F(\mathbf{x})\right|
$$

In one dimension: Kolmogorov-Smirnov statistic

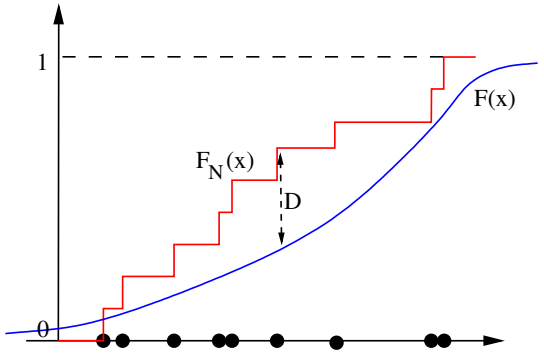


On the convergence of quasi-Random Sampling Importance Resampling

Quasi-Monte Carlo tools

\section{cdf-rep-points}

What set of points is the best representation for $F(\mathbf{x})$ ? 


\section{cdf-rep-points}

What set of points is the best representation for $F(\mathbf{x})$ ?

Definition (cdf-rep-points, [Wang \& Fang, 1990])

A point set $\mathcal{P}^{*}=\left\{\mathbf{x}_{1}, \ldots, \mathbf{x}_{N}\right\}$ for which

$$
D_{N}^{*}\left(\mathcal{P}^{*}, F\right)=\min _{\mathcal{P}} D_{N}^{*}(\mathcal{P}, F),
$$

is called a set of cdf-rep-points of $F(\mathbf{x})$. 


\section{Koksma-Hlawka: a variant}

- Note that the Koksma-Hlawka inequality is traditionally given over the unit s-dimensional hypercube. 


\section{Koksma-Hlawka: a variant}

- Note that the Koksma-Hlawka inequality is traditionally given over the unit s-dimensional hypercube.

- The following variant is over $\mathbb{R}^{s}$ [Chelson, 1976]:

$$
\left|\frac{1}{N} \sum_{n=1}^{N} f\left(\mathbf{x}_{n}\right)-\int_{\mathbb{R}^{s}} f(\mathbf{x}) d G(\mathbf{x})\right| \leq V_{H K}(f) D_{N}^{*}(\mathcal{P}, G) .
$$

$\Rightarrow$ This variant seems less cited and used by MC and QMCpeople! 


\section{Discrepancy-preserving transformation}

- Let $\mathcal{A}=\left\{\mathbf{x}_{1}, \ldots, \mathbf{x}_{N}\right\}$ be a point set in $[0,1]^{s}$.

- Let $G$ be a multivariate CDF having independent marginals:

$$
G(\mathbf{x})=G\left(x_{1}, \ldots, x_{s}\right)=\prod_{i=1}^{s} G_{i}\left(x_{i}\right) .
$$




\section{Discrepancy-preserving transformation}

- Let $\mathcal{A}=\left\{\mathbf{x}_{1}, \ldots, \mathbf{x}_{N}\right\}$ be a point set in $[0,1]^{s}$.

- Let $G$ be a multivariate CDF having independent marginals:

$$
G(\mathbf{x})=G\left(x_{1}, \ldots, x_{s}\right)=\prod_{i=1}^{s} G_{i}\left(x_{i}\right) .
$$

\section{Lemma}

If we take $\mathcal{B}=\left\{\mathbf{y}_{1}, \ldots, \mathbf{y}_{N}\right\}$ as

$$
\mathbf{y}_{k}=\left(G_{1}^{-1}\left(x_{k, 1}\right), \ldots, G_{s}^{-1}\left(x_{k, s}\right)\right), \quad k=1, \ldots, N,
$$

then $D_{N}^{*}(\mathcal{B}, G)=D_{N}^{*}(\mathcal{A})$. 


\section{Discrepancy-preserving transformation}

\section{Corollary}

Let $F(x)$ be a univariate continuous CDF. Then the set

$$
\mathcal{Z}=\left\{z_{i}=F^{-1}\left(\frac{2 i-1}{2 N}\right), i=1, \ldots, N\right\},
$$

contains the cdf-rep-points of size $N$ of $F(x)$ with $D_{N}^{*}(\mathcal{Z}, F)$ optimal and equal to $\frac{1}{2 N}$. 


\section{Discrepancy-preserving transformation}

\section{Corollary}

Let $F(x)$ be a univariate continuous $C D F$. Then the set

$$
\mathcal{Z}=\left\{z_{i}=F^{-1}\left(\frac{2 i-1}{2 N}\right), i=1, \ldots, N\right\},
$$

contains the cdf-rep-points of size $N$ of $F(x)$ with $D_{N}^{*}(\mathcal{Z}, F)$ optimal and equal to $\frac{1}{2 N}$.

- How about the discrete case?

- Let's first review multinomial resampling... 


\section{Multinomial Resampling}

\section{What do we have?}

An ECDF $F_{N}(\theta)$ formed by the samples $\boldsymbol{\theta}_{1}, \ldots, \boldsymbol{\theta}_{N}$ and their respective weights $q_{1}, \ldots, q_{N}$. 


\section{Multinomial Resampling}

What do we have?

An ECDF $F_{N}(\boldsymbol{\theta})$ formed by the samples $\boldsymbol{\theta}_{1}, \ldots, \boldsymbol{\theta}_{N}$ and their respective weights $q_{1}, \ldots, q_{N}$.

What do we want?

$n$ new samples from this distribution (with weights $\frac{1}{n}$ ). 
On the convergence of quasi-Random Sampling Importance Resampling

Quasi-Monte Carlo tools

\section{Multinomial Resampling: 1 dimension}

In one dimension, this is easy:

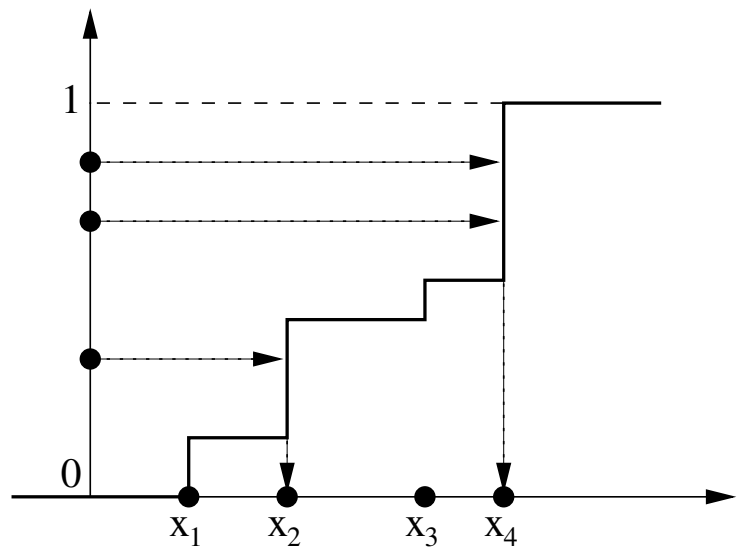




\section{Multinomial Resampling: more than 1 dimension}

In more than one dimension, work with the indices:

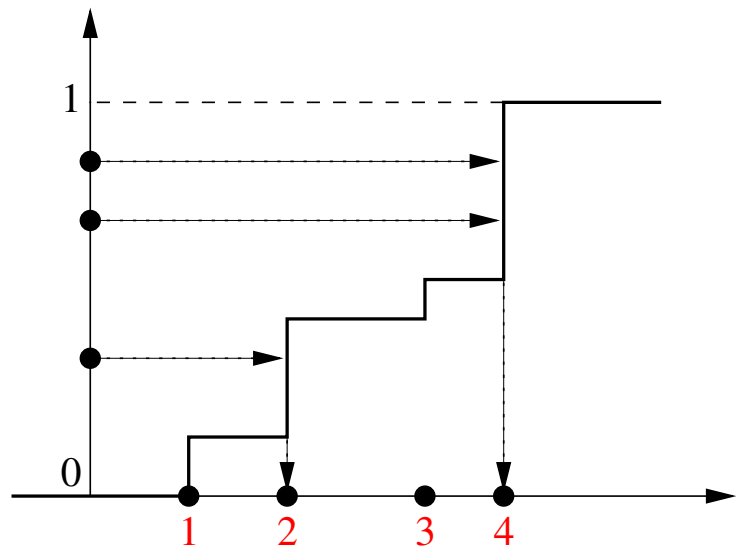




\section{Multinomial resampling}

Note that

- we start from one-dimensional points in $[0,1]$.

- the new samples are all given the same weight $\frac{1}{n}$.

- the new samples of course also have an ECDF.

$\Rightarrow$ Denote this new ECDF by $F_{N, n}(\boldsymbol{\theta})$. 


\section{Multinomial resampling}

What about

$$
\sup _{\boldsymbol{\theta} \in \mathbb{R}^{s}}\left|F_{N}(\boldsymbol{\theta})-F_{N, n}(\boldsymbol{\theta})\right| ?
$$

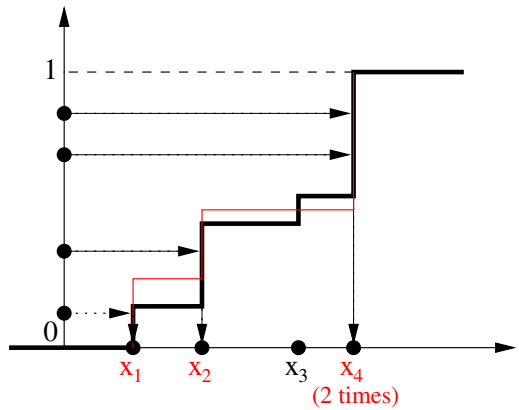

$\Rightarrow$ need to make distinction between 'ordered' and 'unordered' points. 


\section{Ordered vs. unordered points}

The points:
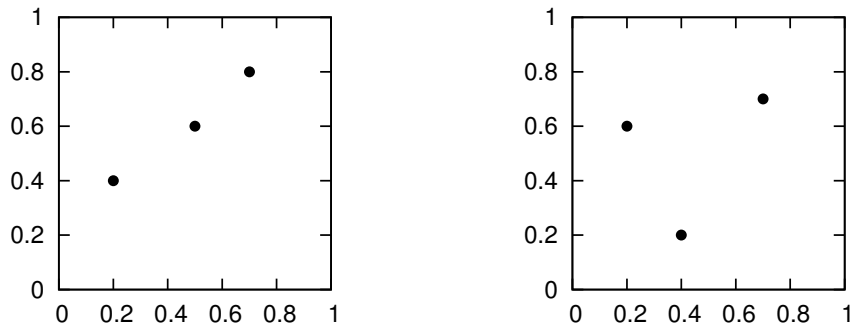

(a) Ordered

(b) Unordered 


\section{Ordered vs. unordered points}

The ECDF's:

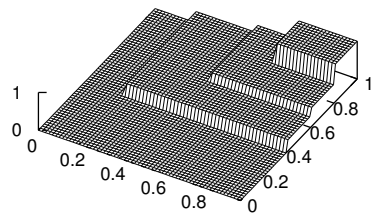

(c) Ordered

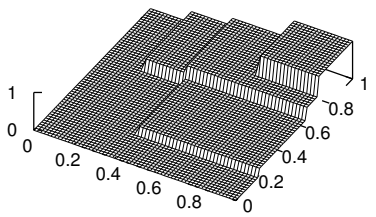

(d) Unordered 


\section{Ordered vs. unordered points}

Having noticed the difference between

$$
\text { ordered vs. unordered }
$$

multidimensional point sets, what can we then say about

$$
\sup _{\boldsymbol{\theta} \in \mathbb{R}^{s}}\left|F_{N}(\boldsymbol{\theta})-F_{N, n}(\boldsymbol{\theta})\right|
$$

if we use the one-dimensional point set

$$
\mathcal{Z}=\left\{z_{i}=\frac{2 i-1}{2 n}, i=1, \ldots, n\right\}
$$

for the multinomial resampling? 
On the convergence of quasi-Random Sampling Importance Resampling

Quasi-Monte Carlo tools

\section{Ordered points}

Let $F_{N}(\boldsymbol{\theta})$ be the ECDF of the ordered $s$-dimensional points $\mathbf{x}_{1} \leq \mathbf{x}_{2} \leq \cdots \leq \mathbf{x}_{N}$ with their respective weights $q_{i}$. 


\section{Ordered points}

Let $F_{N}(\theta)$ be the ECDF of the ordered $s$-dimensional points $\mathbf{x}_{1} \leq \mathbf{x}_{2} \leq \cdots \leq \mathbf{x}_{N}$ with their respective weights $q_{i}$.

\section{Theorem}

If we use the point set

$$
\mathcal{Z}=\left\{z_{i}=\frac{2 i-1}{2 n}, i=1, \ldots, n\right\},
$$

for the multinomial resampling, then

$$
\sup _{\boldsymbol{\theta} \in \mathbb{R}^{s}}\left|F_{N}(\boldsymbol{\theta})-F_{N, n}(\boldsymbol{\theta})\right|<\frac{1}{2 n} .
$$


On the convergence of quasi-Random Sampling Importance Resampling

Quasi-Monte Carlo tools

\section{Ordered points}

One-dimensional example: always ordered!

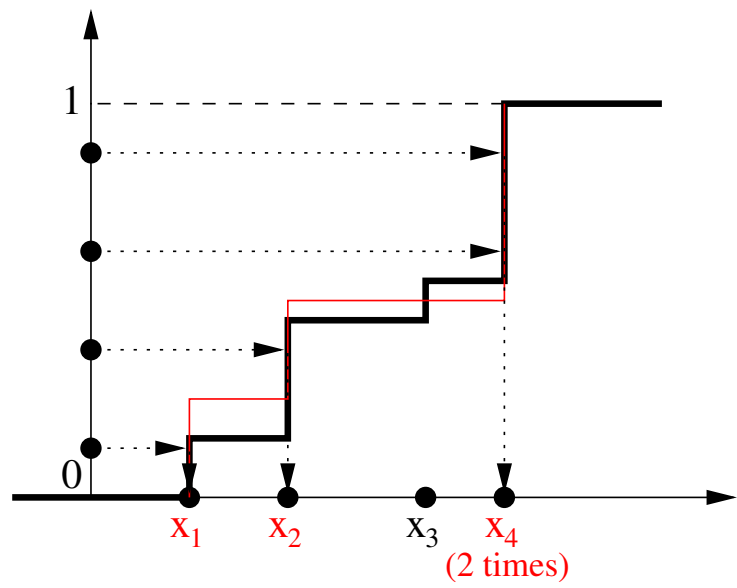




\section{Unordered points}

Let $F_{N}(\boldsymbol{\theta})$ be the ECDF of the unordered $s$-dimensional points $\mathbf{x}_{1} \leq \mathbf{x}_{2} \leq \cdots \leq \mathbf{x}_{N}$ with their respective weights $q_{i}$. 


\section{Unordered points}

Let $F_{N}(\boldsymbol{\theta})$ be the ECDF of the unordered $s$-dimensional points $\mathbf{x}_{1} \leq \mathbf{x}_{2} \leq \cdots \leq \mathbf{x}_{N}$ with their respective weights $q_{i}$.

\section{Theorem}

If we use the point set

$$
\mathcal{Z}=\left\{z_{i}=\frac{2 i-1}{2 n}, i=1, \ldots, n\right\},
$$

for the multinomial resampling, then

$$
\sup _{\boldsymbol{\theta} \in \mathbb{R}^{s}}\left|F_{N}(\boldsymbol{\theta})-F_{N, n}(\boldsymbol{\theta})\right|<\frac{N}{n} .
$$


On the convergence of quasi-Random Sampling Importance Resampling

The SIR and QSIR algorithm

\section{Outline}

\section{(1) Introduction}

(2) Quasi-Monte Carlo tools

(3) The SIR and QSIR algorithm

(4) Numerical Example 


\section{SIR}

Remember the problem:

$$
f(\boldsymbol{\theta})=\frac{h(\boldsymbol{\theta})}{\int_{\mathbb{R}^{s}} h(\boldsymbol{\theta}) d \boldsymbol{\theta}}
$$

A possible solution:

- SIR = Sampling Importance Resampling [Rubin, D. B., 1988].

- Also sometimes called 'Importance Resampling' or 'weighted bootstrap'. 
On the convergence of quasi-Random Sampling Importance Resampling

The SIR and QSIR algorithm

\section{SIR-Algorithm}

\section{Algorithm (SIR-Algorithm)}

(1) Choose a proposal distribution with density $g$. 


\section{SIR-Algorithm}

\section{Algorithm (SIR-Algorithm)}

(1) Choose a proposal distribution with density $g$.

(2) Sample $\boldsymbol{\theta}_{1}, \ldots, \boldsymbol{\theta}_{N}$ from this proposal distribution. 


\section{SIR-Algorithm}

\section{Algorithm (SIR-Algorithm)}

(1) Choose a proposal distribution with density $g$.

(2) Sample $\boldsymbol{\theta}_{1}, \ldots, \boldsymbol{\theta}_{N}$ from this proposal distribution.

(3) Define weights

$$
\omega_{i}=\frac{h\left(\boldsymbol{\theta}_{i}\right)}{g\left(\boldsymbol{\theta}_{i}\right)} \stackrel{\text { normalize }}{\longrightarrow} \quad q_{i}=\frac{\omega_{i}}{\sum_{i=1}^{N} \omega_{i}}, \quad \text { for } i=1, \ldots, N .
$$




\section{SIR-Algorithm}

\section{Algorithm (SIR-Algorithm)}

(1) Choose a proposal distribution with density $g$.

(2) Sample $\boldsymbol{\theta}_{1}, \ldots, \boldsymbol{\theta}_{N}$ from this proposal distribution.

(3) Define weights

$$
\omega_{i}=\frac{h\left(\boldsymbol{\theta}_{i}\right)}{g\left(\boldsymbol{\theta}_{i}\right)} \stackrel{\text { normalize }}{\longrightarrow} \quad q_{i}=\frac{\omega_{i}}{\sum_{i=1}^{N} \omega_{i}}, \quad \text { for } i=1, \ldots, N .
$$

(3) generate $n$ samples $\boldsymbol{\theta}^{*}$ from the ECDF of $\left\{\boldsymbol{\theta}_{1}, \ldots, \boldsymbol{\theta}_{N}\right\}$ with mass $q_{i}$ at $\boldsymbol{\theta}_{i}$. 


\section{SIR-Algorithm}

\section{Algorithm (SIR-Algorithm)}

(1) Choose a proposal distribution with density $g$.

(2) Sample $\boldsymbol{\theta}_{1}, \ldots, \boldsymbol{\theta}_{N}$ from this proposal distribution.

(3) Define weights

$$
\omega_{i}=\frac{h\left(\boldsymbol{\theta}_{i}\right)}{g\left(\boldsymbol{\theta}_{i}\right)} \stackrel{\text { normalize }}{\longrightarrow} \quad q_{i}=\frac{\omega_{i}}{\sum_{i=1}^{N} \omega_{i}}, \quad \text { for } i=1, \ldots, N .
$$

(3) generate $n$ samples $\boldsymbol{\theta}^{*}$ from the ECDF of $\left\{\boldsymbol{\theta}_{1}, \ldots, \boldsymbol{\theta}_{N}\right\}$ with mass $q_{i}$ at $\boldsymbol{\theta}_{i}$.

We then have

$$
\boldsymbol{\theta}^{*} \sim f(\boldsymbol{\theta})
$$




\section{Restriction}

For the rest of the talk, $G$ is a multivariate CDF having independent marginals:

$$
G(\mathbf{x})=G\left(x_{1}, \ldots, x_{s}\right)=\prod_{i=1}^{s} G_{i}\left(x_{i}\right),
$$




\section{QSIR}

QSIR = Quasi-random Sampling Importance Resampling [Pérez et al., 2005]

- Two modifications of the original SIR

- Based on Quasi-Monte Carlo ideas

- Empirically verified to be better:

- samples have smaller F-discrepancy

- samples are thus 'more representative' 


\section{QSIR: Algorithm 1}

The first modification:

\section{Algorithm (QSIR 1)}

(1) Generate a quasi-random sample $\boldsymbol{\theta}_{1}, \ldots, \boldsymbol{\theta}_{N}$ from the density $g$. 


\section{QSIR: Algorithm 1}

The first modification:

\section{Algorithm (QSIR 1)}

(1) Generate a quasi-random sample $\boldsymbol{\theta}_{1}, \ldots, \boldsymbol{\theta}_{N}$ from the density $g$.

(2) Calculate the weights $q_{i}$. 


\section{QSIR: Algorithm 1}

The first modification:

\section{Algorithm (QSIR 1)}

(1) Generate a quasi-random sample $\boldsymbol{\theta}_{1}, \ldots, \boldsymbol{\theta}_{N}$ from the density $g$.

(2) Calculate the weights $q_{i}$.

(3) Obtain a random sample of size $n$ from the ECDF of $\left\{\boldsymbol{\theta}_{1}, \ldots, \boldsymbol{\theta}_{N}\right\}$ with mass $q_{i}$ at $\boldsymbol{\theta}_{\mathbf{i}}$. 


\section{QSIR: Algorithm 1}

The first modification:

\section{Algorithm (QSIR 1)}

(1) Generate a quasi-random sample $\boldsymbol{\theta}_{1}, \ldots, \boldsymbol{\theta}_{N}$ from the density $g$.

(2) Calculate the weights $q_{i}$.

(3) Obtain a random sample of size $n$ from the ECDF of $\left\{\boldsymbol{\theta}_{1}, \ldots, \boldsymbol{\theta}_{N}\right\}$ with mass $q_{i}$ at $\boldsymbol{\theta}_{\mathbf{i}}$.

$\Rightarrow$ quasi-randomness in the first (sample) step.

$\Rightarrow$ randomness in the last (resample) step. 


\section{QSIR: Algorithm 2}

The second modification:

\section{Algorithm (QSIR 2)}

(1) Generate a quasi-random sample $\boldsymbol{\theta}_{1}, \ldots, \boldsymbol{\theta}_{N}$ from the density $g$. 


\section{QSIR: Algorithm 2}

The second modification:

\section{Algorithm (QSIR 2)}

(1) Generate a quasi-random sample $\boldsymbol{\theta}_{1}, \ldots, \boldsymbol{\theta}_{N}$ from the density $g$.

(2) Calculate the weights $q_{i}$. 


\section{QSIR: Algorithm 2}

The second modification:

\section{Algorithm (QSIR 2)}

(1) Generate a quasi-random sample $\boldsymbol{\theta}_{1}, \ldots, \boldsymbol{\theta}_{N}$ from the density $g$.

(2) Calculate the weights $q_{i}$.

(3) Calculate $\left\{u_{i}=\frac{2 i-1}{2 n}, i=1, \ldots, n\right\}$ for a sample size $n$. 


\section{QSIR: Algorithm 2}

The second modification:

\section{Algorithm (QSIR 2)}

(1) Generate a quasi-random sample $\boldsymbol{\theta}_{1}, \ldots, \boldsymbol{\theta}_{N}$ from the density $g$.

(2) Calculate the weights $q_{i}$.

(3) Calculate $\left\{u_{i}=\frac{2 i-1}{2 n}, i=1, \ldots, n\right\}$ for a sample size $n$.

(3) Use $\left\{u_{1}, \ldots, u_{n}\right\}$ to obtain a sample of size $n$ from the the ECDF which places mass $q_{i}$ at $\boldsymbol{\theta}_{\mathbf{i}}$. 


\section{QSIR: Algorithm 2}

The second modification:

\section{Algorithm (QSIR 2)}

(1) Generate a quasi-random sample $\boldsymbol{\theta}_{1}, \ldots, \boldsymbol{\theta}_{N}$ from the density $g$.

(2) Calculate the weights $q_{i}$.

(3) Calculate $\left\{u_{i}=\frac{2 i-1}{2 n}, i=1, \ldots, n\right\}$ for a sample size $n$.

(3) Use $\left\{u_{1}, \ldots, u_{n}\right\}$ to obtain a sample of size $n$ from the the ECDF which places mass $q_{i}$ at $\boldsymbol{\theta}_{\mathbf{i}}$.

$\Rightarrow$ quasi-randomness in both sample and resample step.

$\Rightarrow$ completely deterministic! 


\section{Algorithm 1 and 2: convergence after first step}

For the first step of both algorithms,

- we start from a QMC-point set $\mathcal{L}$ on $[0,1]^{\text {s }}$ of size $N$ with star discrepancy $D_{N}^{*}(\mathcal{L})$.

- $G$ is assumed to define a discrepancy-preserving transformation.

Therefore... 


\section{Algorithm 1 and 2: convergence after first step}

For the first step of both algorithms,

- we start from a QMC-point set $\mathcal{L}$ on $[0,1]^{\text {s }}$ of size $N$ with star discrepancy $D_{N}^{*}(\mathcal{L})$.

- $G$ is assumed to define a discrepancy-preserving transformation.

Therefore...

Theorem ( $G$-discrepancy after step 1 )

The $G$-discrepancy of the samples $\mathcal{P}=\left\{\boldsymbol{\theta}_{1}, \ldots, \boldsymbol{\theta}_{N}\right\}$ has the value $D_{N}^{*}(\mathcal{L})$. 


\section{Algorithm 1 and 2: convergence after first step (1D)}

In one dimension, since both algorithms start from

$$
\mathcal{L}=\left\{\frac{2 i-1}{2 N}, i=1, \ldots, N\right\}
$$

with best possible discrepancy $\frac{1}{2 N}$, we have... 


\section{Algorithm 1 and 2: convergence after first step (1D)}

In one dimension, since both algorithms start from

$$
\mathcal{L}=\left\{\frac{2 i-1}{2 N}, i=1, \ldots, N\right\}
$$

with best possible discrepancy $\frac{1}{2 N}$, we have...

Corollary ( $G$-discrepancy after step 1 in one dimension)

The $G$-discrepancy of the samples $\theta_{i}$ will have the optimal value $\frac{1}{2 N}$. 


\section{Algorithm 1: convergence after second step}

After the second step (the multinomial random resampling) we have

Theorem (Algorithm 1)

$$
\sup _{\boldsymbol{\theta} \in \mathbb{R}^{s}}\left|F(\boldsymbol{\theta})-F_{N, n}(\boldsymbol{\theta})\right| \in \mathcal{O}\left(\max \left(\kappa(N), \frac{1}{\sqrt{n}}\right)\right) .
$$

with

$$
\kappa(N)= \begin{cases}\frac{1}{N} & \text { if } s=1, \\ \frac{(\log (N))^{s}}{N} & \text { if } s>1 .\end{cases}
$$




\section{Algorithm 2: convergence after second step}

After the second step (the multinomial deterministic resampling) we have

Theorem (Algorithm 2)

$$
\sup _{\boldsymbol{\theta} \in \mathbb{R}^{s}}\left|F_{N, n}(\boldsymbol{\theta})-F(\boldsymbol{\theta})\right| \leq \tau(N, n)+\mathcal{O}(\kappa(N)) .
$$

with

$$
\tau(N, n)= \begin{cases}\frac{1}{2 n} & \text { if } s=1 \\ \frac{N}{n} & \text { if } s>1\end{cases}
$$

and

$$
\kappa(N)= \begin{cases}\frac{1}{N} & \text { if } s=1, \\ \frac{(\log (N))^{s}}{N} & \text { if } s>1 .\end{cases}
$$




\section{Convergence summarized}

Summarized:

\begin{tabular}{|cc|c|c|}
\hline & & $s=1$ & $s>1$ \\
\hline Alg. 1 & ordered & $\mathcal{O}\left(\max \left(\frac{1}{N}, \frac{1}{\sqrt{n}}\right)\right)$ & $\mathcal{O}\left(\max \left(\frac{(\log N)^{s}}{N}, \frac{1}{\sqrt{n}}\right)\right)$ \\
& unordered & $/$ & $\mathcal{O}\left(\max \left(\frac{(\log N)^{s}}{N}, \frac{1}{\sqrt{n}}\right)\right)$ \\
\hline Alg. 2 & ordered & $\frac{1}{2 n}+\mathcal{O}\left(\frac{1}{N}\right)$ & $\frac{1}{2 n}+\mathcal{O}\left(\frac{(\log N)^{s}}{N}\right)$ \\
& unordered & $/$ & $\frac{N}{n}+\mathcal{O}\left(\frac{(\log N)^{s}}{N}\right)$ \\
\hline
\end{tabular}

$\Rightarrow$ relative sizes of $n$ and $N$ matter! 
On the convergence of quasi-Random Sampling Importance Resampling

Numerical Example

\section{Outline}

\section{(1) Introduction}

(2) Quasi-Monte Carlo tools

(3) The SIR and QSIR algorithm

4) Numerical Example 


\section{Numerical example}

Take $g$ as the standard Cauchy distribution

$$
g(x)=\frac{1}{\pi\left(1+x^{2}\right)}
$$

and $f$ the $t$-distribution with two degrees of freedom

$$
f(x)=\frac{\Gamma(3 / 2)}{\sqrt{2 \pi} \Gamma(1)}\left(1+\frac{x^{2}}{2}\right)^{-3 / 2} .
$$




\section{Numerical example}

$f$ is already normalized, so $f=h$ and we have

$$
\frac{h(x)}{g(x)}=\frac{\pi}{2 \sqrt{2}}\left(1+x^{2}\right)\left(1+\frac{x^{2}}{2}\right)^{-3 / 2}
$$

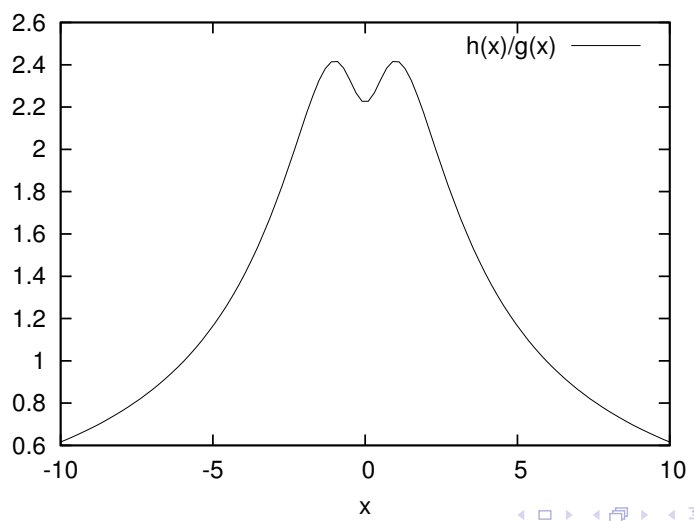




\section{Numerical example: Algorithm 1}

$$
\sup _{\boldsymbol{\theta} \in \mathbb{R}^{s}}\left|F_{N, n}(\boldsymbol{\theta})-F(\boldsymbol{\theta})\right| \in \mathcal{O}\left(\max \left(\frac{1}{N}, \frac{1}{\sqrt{n}}\right)\right)
$$
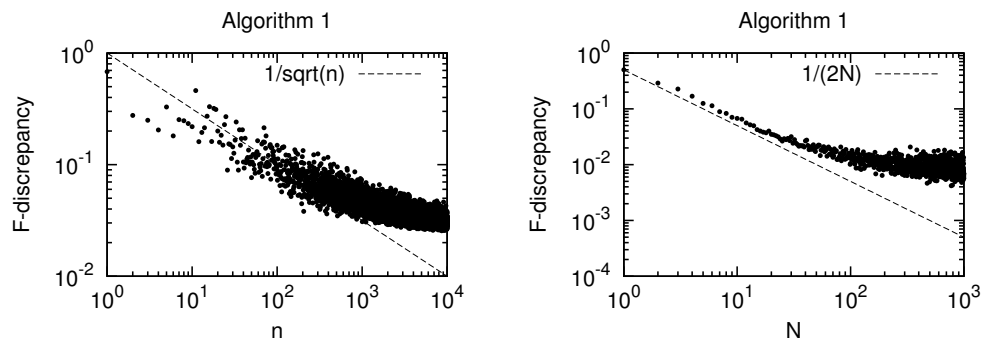

(e) fixed $N$

(f) fixed $n$ 


\section{Numerical example: Algorithm 2}

$$
\sup _{\boldsymbol{\theta} \in \mathbb{R}^{s}}\left|F_{N, n}(\boldsymbol{\theta})-F(\boldsymbol{\theta})\right| \leq \mathcal{O}\left(\frac{1}{N}\right)+\frac{1}{2 n}
$$

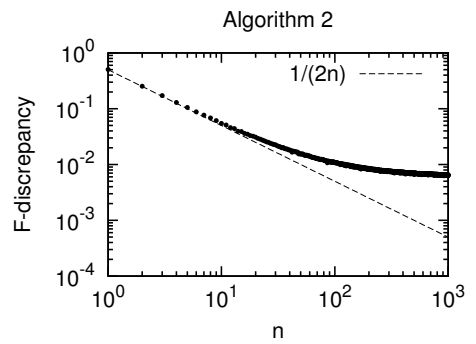

(g) fixed $N$

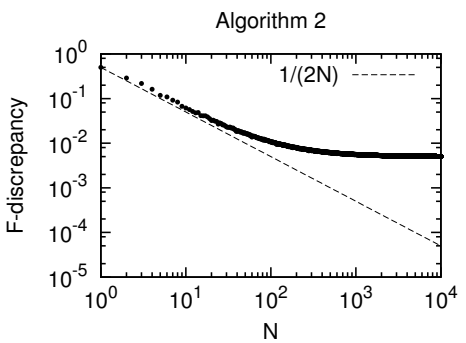

(h) fixed $n$ 
On the convergence of quasi-Random Sampling Importance Resampling

Conclusions

\section{Outline}

\section{(1) Introduction}

(2) Quasi-Monte Carlo tools

(3) The SIR and QSIR algorithm

(4) Numerical Example

(5) Conclusions 
On the convergence of quasi-Random Sampling Importance Resampling

Conclusions

\section{Conclusions}

- Our analysis explains empirical results from [Pérez et al., 2005]. 
On the convergence of quasi-Random Sampling Importance Resampling

Conclusions

\section{Conclusions}

- Our analysis explains empirical results from [Pérez et al., 2005].

- Asymptotic error convergence results for QSIR. 


\section{Conclusions}

- Our analysis explains empirical results from [Pérez et al., 2005].

- Asymptotic error convergence results for QSIR.

- In one dimension, if $N=n$, then Algorithm 1 is $\mathcal{O}(1 / \sqrt{N})$ and Algorithm 2 is $\mathcal{O}(1 / N)$. 


\section{Conclusions}

- Our analysis explains empirical results from [Pérez et al., 2005].

- Asymptotic error convergence results for QSIR.

- In one dimension, if $N=n$, then Algorithm 1 is $\mathcal{O}(1 / \sqrt{N})$ and Algorithm 2 is $\mathcal{O}(1 / N)$.

- The order of convergence depends on $s, N$, and $n$ and their relative sizes influence the usefulness of the bounds ( $F$-discrepancy is never larger than 1 ). 


\section{Conclusions}

- Our analysis explains empirical results from [Pérez et al., 2005].

- Asymptotic error convergence results for QSIR.

- In one dimension, if $N=n$, then Algorithm 1 is $\mathcal{O}(1 / \sqrt{N})$ and Algorithm 2 is $\mathcal{O}(1 / N)$.

- The order of convergence depends on $s, N$, and $n$ and their relative sizes influence the usefulness of the bounds ( $F$-discrepancy is never larger than 1$)$.

- Instead of a QMC-point set for step 1, one could use $\left\{u_{i}=\frac{2 i-1}{2 n}\right\}$ for each dimension... 


\section{Conclusions}

- Our analysis explains empirical results from [Pérez et al., 2005].

- Asymptotic error convergence results for QSIR.

- In one dimension, if $N=n$, then Algorithm 1 is $\mathcal{O}(1 / \sqrt{N})$ and Algorithm 2 is $\mathcal{O}(1 / N)$.

- The order of convergence depends on $s, N$, and $n$ and their relative sizes influence the usefulness of the bounds ( $F$-discrepancy is never larger than 1 ).

- Instead of a QMC-point set for step 1, one could use $\left\{u_{i}=\frac{2 i-1}{2 n}\right\}$ for each dimension...

- Disadvantage: the term $\mathcal{O}\left(\frac{(\log N)^{s}}{N}\right)$ becomes worse. 


\section{Conclusions}

- Our analysis explains empirical results from [Pérez et al., 2005].

- Asymptotic error convergence results for QSIR.

- In one dimension, if $N=n$, then Algorithm 1 is $\mathcal{O}(1 / \sqrt{N})$ and Algorithm 2 is $\mathcal{O}(1 / N)$.

- The order of convergence depends on $s, N$, and $n$ and their relative sizes influence the usefulness of the bounds ( $F$-discrepancy is never larger than 1 ).

- Instead of a QMC-point set for step 1, one could use $\left\{u_{i}=\frac{2 i-1}{2 n}\right\}$ for each dimension...

- Disadvantage: the term $\mathcal{O}\left(\frac{(\log N)^{s}}{N}\right)$ becomes worse.

- Advantage: the samples will be ordered, so for Algorithm 2, the term $\frac{N}{n}$ can be replaced by $\frac{1}{2 n}$. 
Thank you for your attention!

Questions? Suggestions?

Bart.Vandewoestyne@cs.kuleuven.be 